\title{
ELECTRICAL AND SPECTROSCOPIC DIAGNOSTICS OF ATMOSPHERIC PRESSURE DBD PLASMA JET
}

\author{
N. C. ROY* and M. R. TALUKDER \\ Plasma Science and Technology Laboratory, Department of Applied Physics and \\ Electronic Engineering, University of Rajshahi, Rajshahi-6205, Bangladesh
}

\begin{abstract}
Atmospheric pressure capillary dielectric barrier oxygen discharge plasma jet is developed to generate nonthermal plasma using unipolar positive pulse power supply. Both electrical and optical diagnostic techniques were used to characterize the produced plasma as functions of applied voltage and gas flow rate. Electrical diagnostics indicated that the discharge frequency decreased with gas flow rate but increased with the applied voltage. Analytical results obtained from the optical emission spectroscopic data revealed the gas temperature, excitation temperature and electron density. Gas temperature was found to decrease with increasing oxygen flow rate but increase linearly with applied voltage. The produced plasma was applied preliminarily to study the inactivation yield of Fusarium oxysporum fungus infected potato samples.
\end{abstract}

Key words: Dielectric barrier discharge, Capillary DBD jet, Spectroscopic diagnostics, Oxygen plasma

\section{INTRODUCTION}

Atmospheric pressure dielectric barrier discharge plasma jets (APDJ) have drawn great interest to the researchers in the recent years. Researchers are conducting researches of APDJs in the field of biology (e.g. inactivation of fungus, virus, bacteria, tumour, cancerous cell, etc.), chemistry (e.g. barrier coatings on polymers, carboxyl functionalization, deposition of fluorocarbon films, and so on) (d'Agostino et al. 2005) and surface treatments (e.g. plasma cleaning, plasma sterilization, treatment of fabrics and fibers, and so on) (Roth 2001). The APDJs produce a large number of ions, reactive species and free radicals because the energetic electrons enhance the gas chemistry. APDJs are able to generate nonthermal plasma, with low gas temperature and high electron temperature, in open space rather than in a confined discharge chamber without any arrangement for controlling pressure inside the chamber. Another advantage is that the size and position of the sample to be treated is not a factor and the plasma jet can be applied on the sample directly. Several parameters can control the properties (Zhang et al. 2015, Ionascut-Nedelcescu et al. 2008) of APDJ such as jet geometry, type of

\footnotetext{
* Corresponding author: <neporoy@gmail.com>.
} 
dielectric, excitation frequency, type of power source and gas composition including gas flow rate. Due to low gas temperature, APDJs do not cause thermal damage to heatsensitive biological (Stoffels et al. 2006) systems such as living tissues and cells. These application processes can be enhanced and controlled by better understanding the properties of the produced plasma. Because, the gas flow dynamics are different (Zhang et al. 2015) in each type of jet due to jet structure with nozzle geometry, Reynolds number and initial velocity. The objective of this work was to develop and investigate the properties of the produced plasma jet for biological applications.

Gas temperature $\left(T_{g}\right)$, electron density $\left(n_{e}\right)$ and electron temperature $\left(T_{e}\right)$ are the three fundamental properties of plasmas and have their significant effects on the operation and maintenance of gas discharge. In most biological applications (Fridman et al. 2013) of APDJs, $T_{g}$ has a significant influence (Park et al. 2010) on the sample to be investigated, so that $T_{g}$ needs to diagnose more precisely in order to attain at room temperature and to maintain highly stable for efficient chemical reactions to meet the pertaining requirements. Electrical and optical characterizations are carried out for understanding the properties of the APDJ.

Experimental setup for the production and measurements of APDJ plasma has been presented. Analytical results and discussion concerned with the measurements are also discussed in the text.

\section{MATERIALS AND METHODS}

The schematic diagram of the experimental setup of APDJ is shown in Fig. 1. It consists of a high voltage power source, plasma jet, and oxygen $\left(\mathrm{O}_{2}\right)$ gas cylinder incorporating with a gas flow controller. The voltage, power and frequency of the power supply are approximately $4 \mathrm{kV}, 100 \mathrm{~W}$ and $60 \mathrm{kHz}$, respectively. The power supply can be operated both in unipolar positive and bipolar modes as necessary. The jet is made using pyrex glass tubes. Length, outer and inner diameters of the capillary tube are 15, 1.4 and $0.9 \mathrm{~mm}$, respectively. The inner electrode, made of a $0.50 \mathrm{~mm}$ diameter copper wire, is placed inside the capillary tube. A cylindrical outer electrode, made of copper tube of 14. $\mathrm{mm}$ inner diameter, is placed outside the capillary tube. Spacing between the inner electrode and the inner surface of the capillary tube is maintained at $0.2 \mathrm{~mm}$. The inner and outer electrodes are connected with the high voltage power supply as shown in Fig. 1. The electrodes are arranged such that the cross-electric field can prevail within the electrode gap. Unipolar positive voltage pulse is applied across the electrodes. Our experimental setup is different from that of Thiyagarajan et al. (2013) in that they used linear-field type electrode configuration with bipolar sinusoidal type voltage source having different source parameters. In our setup, commercial grade $\mathrm{O}_{2}$ gas $(99.65 \%)$ is fed through the upper side of the inner glass tube from the $\mathrm{O}_{2}$ gas cylinder. 


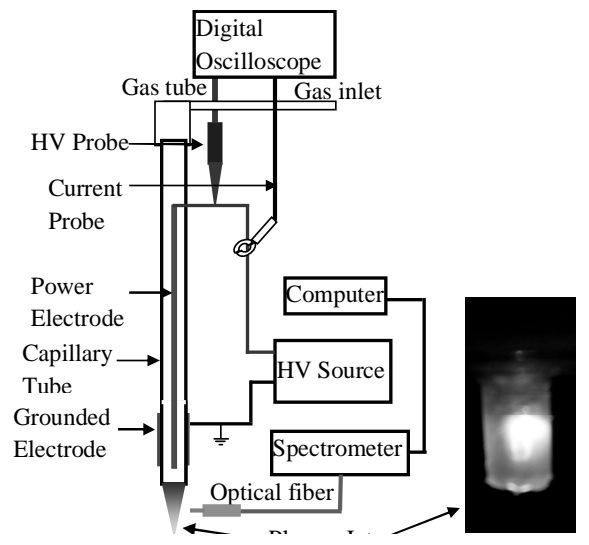

Fig. 1. Schematic of the experimental setup of the atmospheric pressure capillary dielectric barrier discharge oxygen plasma jet.

The flow rate of $O_{2}$ gas is controlled by a gas flow controller (KIT 115P). The voltage across the electrodes is measured with an oscilloscope (GDS 1022) in combination with a high voltage probe $(H V P-08)$. The current flowing through the plasma is measured by a current probe $\left(C P-07^{\circ} \mathrm{C}\right)$.

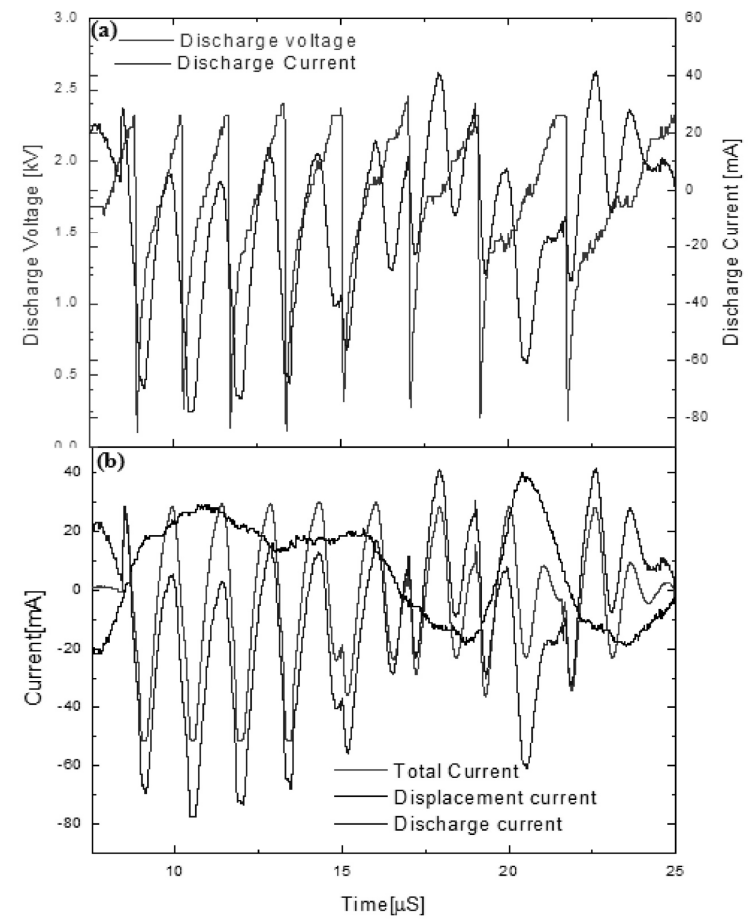

Fig. 2. (a) Discharge voltage and current waveforms of the APDJ plasma, (b) waveform of currents in plasma discharge measured at $2.5 \mathrm{kV}$ with $\mathrm{O}_{2}$ flow rate of $3 \mathrm{lmp}$. 
Emitted spectrum from the produced plasma is fed to the spectrometer (USB $2000+$ $X R$ 1) through a $200 \mathrm{~cm}$ long optical fiber cable. The spectrometer is associated with a computer for spectral data acquisition. At different applied voltages and $\mathrm{O}_{2}$ gas flow rates, the corresponding discharge voltages, currents and emitted spectra are recorded in order to study the properties (Flatin et al. 1999) of the APDJ discharges.

Typical discharge voltage and current waveforms of the APDJ measured at $2.5 \mathrm{kV}$ with $\mathrm{O}_{2}$ flow rate of $3 \mathrm{lpm}$ are shwon in Fig. 2. The recorded emission spectrum along with the identified major peaks of the species is given in Fig. 3. The analytical results are discussed in the following sections.

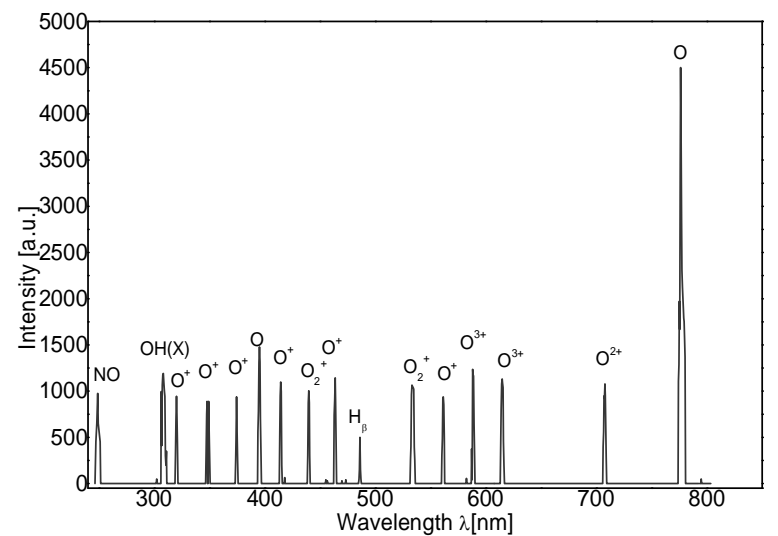

Fig. 3. Emitted spectra recorded at $2.5 \mathrm{kV}$ with $\mathrm{O}_{2}$ flow rate of $3 \mathrm{lmp}$ along with the identified species.

\section{RESULTS AND DISCUSSION}

The voltage is applied to the inner electrode and $\mathrm{O}_{2}$ gas is injected into the capillary jet. With increasing the applied voltage to some extent, breakdown of $\mathrm{O}_{2}$ gas is occurred. In order to observe electrical characteristics of the produced plasma the discharge voltage and current waveforms (Xiao et al. 2014, Ni et al. 2008) are displayed in Fig. 2(a). The discharge current is obtained by using following equation (Rusterholtz et al. 2013)

$$
I_{\text {tot }}=I_{\text {diskp. }}+I_{\text {dis. }}
$$

where $I_{t o t}, I_{\text {diskp. }}$ and $I_{\text {dis. }}$ are total current (with plasma discharge condition), displacement current (without plasma discharge) and discharge current respectively, and their corresponding waveforms are shown in Fig. 2(b).

The production and maintenance of the capillary $\mathrm{O}_{2}$ DBD plasma jet are found to be sensitive to the applied voltage and gas flow rate. The electronegative $\mathrm{O}_{2}$ gas plays a significant role of plasma discharges in air (Raizer et al. 1991). Generally, breakdown of 
$\mathrm{O}_{2}$ gas occurs from several microdischarges that are randomly spread in time and space at atmospheric pressure DBD plasma jet (Thiyagarajan et al. 2013). The DBD plasma is produced with increased $\mathrm{O}_{2}$ gas flow rate but it looks filamentary-like at the flow rate lower than $2 \mathrm{lmp}$. It is seen from Fig. 2 that the discharge current starts to flow when the applied voltage pulse attains a certain voltage level. Energetic electrons, because of gaining kinetic energy from the applied electric field, excite oxygen species to higher energy levels through inelastic collisions during the drifting process of the electrons. When the applied electric field is greater than the critical value of breakdown, electrons move toward the power electrode and hence the electron density increases through electron avalanche process ( $\mathrm{Li}$ et al. 2013). However, the discharge current flows as soon as the conduction channel is formed in the discharge gap and hence the discharge voltage approaches near zero across the electrode gap. This process repeats in each cycle of the discharge voltage pulse.

It was observed during experiment that the plasma discharge volume increases with growing $\mathrm{O}_{2}$ gas flow rate. The discharges appear intense within the electrode space and much weaker outside the electrode region. This occurs in such a cross-field capillary DBD jet plasma discharge due to the monotonic decay of electric field results from spatially confined gas ionization in the electrode region. The applied voltage with rapid oscillation provides radially directed momentum to the electrons, and hence it is difficult for electrons to be transported axially toward the downstream (Walsh and Kong 2008) of the capillary tube.

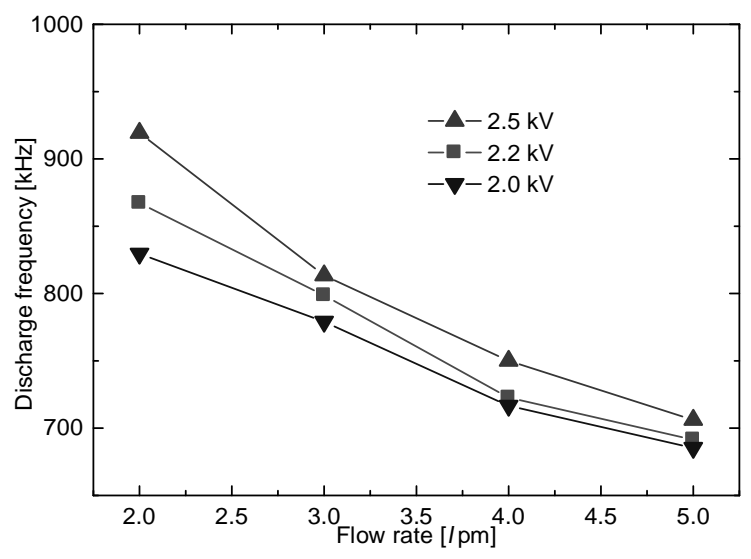

Fig. 4. Dependence of discharge frequency on $\mathrm{O}_{2}$ gas flow rate as a function of voltage.

The operating frequency plays a significant role for the formation and maintenance of the discharge at atmospheric pressure DBD plasma jet. Fig. 4 shows the effect of gas flow rate and applied voltage on the discharge frequency. The discharge frequency 
spectrum is determined (Arkhipenko et al. 2013) by taking the Fourier transformation of the discharge current. The frequency spectrum indicates that the atmospheric pressure DBD plasma consists of many micro-discharges as mentioned earlier. However, in the plot, it is taken into account only that frequency which contains highest amplitude. The discharge frequency of the produced plasma changes with the change in applied voltage and gas flow rate. The drifting velocity of the spatial charges is related to the electric field and hence the spatial charges are accumulated to the power electrode. With increasing electric field, the drifting velocity of the spatial charges is also increased and hence the discharge frequency increases with the increase of applied voltage (Li et al. 2013). This phenomenon is counteracting the increased $O_{2}$ gas flow rate with discharge frequency. This can be attributed with the Reynolds number $R_{e}=n_{g} v_{g} l_{c} / \mu_{d}$, where $n_{g}, v_{g}, l_{c}$ and $\mu_{d}$ are the gas density, gas velocity, characteristic length and dynamic viscosity, respectively. The Reynolds number increases with increasing $\mathrm{O}_{2}$ gas flow rate (Zhang et at. 2015). It is seen from the above relation that the density of $\mathrm{O}_{2}$ gas at the exit of the jet nozzle increases with increasing the Reynolds number and hence the electric field threshold for $\mathrm{O}_{2}$ gas breakdown increases. As a result, the discharge frequency decreases with increasing $\mathrm{O}_{2}$ gas flow rate.

For plasma diagnostics, Langmuir probe at atmospheric pressure (Talukder et al. 2002) provides better accuracy. But, due to small plasma volume and the structure of the jet in present experiment, the probe technique is not suitable for the diagnostics of the produced plasma. Hence, the optical emission spectroscopic (OES) technique is applied to analyze the properties of plasma at atmospheric pressure. The basis of OES data analyses for the determination of $T_{g}$ and $n_{e}$ are the line broadenings due to Doppler and Stark effects. While the electronic excitation temperature $\left(T_{\text {exc }}\right)$ is determined using Boltzmann plot method. In order to have an in-depth understanding of APDJ, characteristics of the discharges are studied as function of voltage applied and gas flow rate to find out suitable experimental conditions for the expected biological application.

The species produced in the plasma are identified using the NIST database and the plasma parameters are estimated analyzing the spectra. In Fig. 3, a typical OES spectrum, measured at $2.5 \mathrm{kV}$ with $\mathrm{O}_{2}$ flow rate of $3 \mathrm{lpm}$ in air, is presented. The dominant peaks observed in the spectra are $\mathrm{NO}\left(\mathrm{B}^{2} \Pi-\mathrm{X}^{2} \Pi\right)$ at $248.78 \mathrm{~nm}, \mathrm{OH}\left(X^{2} \Pi\left(v^{\prime}=0\right) \rightarrow A^{2} \Sigma^{+}\left(v^{\prime \prime}=\right.\right.$ $0)$ at $308.17, \mathrm{O}^{+}\left(\left({ }^{3} P\right) 5 f-\left({ }^{3} P\right) 3 d\right)$ at $\left.320.06 \mathrm{~nm}, \mathrm{O}^{+}\left({ }^{3} P\right) 3 d-\left({ }^{3} P\right) 3 p\right)$ at $348.59 \mathrm{~nm}, O^{+}$ $\left(\left({ }^{3} P\right) 3 d-\left({ }^{3} P\right) 3 p\right)$ at $\left.374.42 \mathrm{~nm}, \mathrm{O}\left({ }^{4} S^{\circ}\right) 3 s-\left({ }^{4} P^{\circ}\right) 3 p\right)$ at $395.19 \mathrm{~nm}, O^{+}\left(\left({ }^{5} S^{\circ}\right) 3 d-\left({ }^{5} S^{\circ}\right) 3 p\right)$ at $414.37 \mathrm{~nm}, O_{2}^{+}\left(A^{2} \Pi_{u}\left(v^{\prime \prime}=11 \rightarrow X^{2} \Pi_{g}\left(v^{\prime}=2\right)\right)\right.$ at $439.80 \mathrm{~nm}, H_{\beta}(4 d-2 p)$ at 486.12 $\mathrm{nm}, O^{+}\left(\left({ }^{4} S^{\circ}\right) 3 d-\left({ }^{4} S^{\circ}\right) 3 p\right)$ at $533.07 \mathrm{~nm}, O^{+}\left(\left({ }^{1} S\right) 3 p-\left({ }^{3} P\right) 3 s\right)$ at $561.10 \mathrm{~nm}, O^{3+}\left(\left({ }^{3} P\right) 3 d-\right.$ $\left.\left({ }^{3} P\right) 3 p\right)$ at $588.24 \mathrm{~nm}, O^{3+}\left(\left({ }^{3} P\right) 3 d-\left({ }^{3} P^{\circ}\right) 3 s\right)$ at $614.23 \mathrm{~nm}, O^{2+}\left(\left({ }^{2} P^{\circ}\right) 4 d-\left({ }^{2} P^{\circ}\right) 4 p\right)$ at $707.91 \mathrm{~nm}$ and $O\left(\left({ }^{5} P\right) 3 p-\left({ }^{5} S\right) 3 s\right)$ at $776.06 \mathrm{~nm}$. The emission from $\mathrm{OH}$ radical can be 
originated from the fragmentation of water molecules present in the air. It is to be noted that $\mathrm{O}_{2}$ is used as working gas for plasma production in open air. The emission from NO is also produced due to the interactions of the plasma species concerned with the air molecules. It is found from Fig. 3 that the emission lines of $O$ atoms at $395.19 \mathrm{~nm}$ and $776.06 \mathrm{~nm}$ contain high intensity in the spectra. This indicates that the density of $O$ atoms is much higher than any other species observed. Because the $O$ atoms are produced dominantly through collisions R2, R4 and R5, as mentioned in Table 1, due to their high production rate coefficients (Lieberman et al. 1994, Moravej et al. 2006) with other species involved in the collision processes.

Table 1. Dominant collision processes in capillary DBD oxygen plasma jet with their rate constants.

\begin{tabular}{rlll}
\hline No. & Reactions & Rate constant $\left(\mathrm{cm}^{3} / \mathrm{s}\right)$ & Ref. \\
\hline (R1) & $e+O_{2}+$ momentum transfer & $4.7 \times 10^{-8} T_{e}^{0.5}$ & {$[19]$} \\
(R2) & $e+O_{2} \rightarrow 2 O+e$ & $4.2 \times 10^{-9} \exp \left(-5.6 / T_{e}\right)$ & {$[19]$} \\
(R3) & $e+O_{2} \rightarrow O_{2}^{+}+2 e$ & $9.0 \times 10^{-10} T_{e}^{0.5} \exp \left(-12.6 / T_{e}\right)$ & {$[19]$} \\
(R4) & $e+O_{2} \rightarrow O^{+}+O+2 e$ & $5.3 \times 10^{-10} T_{e}^{0.9} \exp \left(-20 / T_{e}\right)$ & {$[19]$} \\
(R5) & $e+O_{2}^{+} \rightarrow 2 O$ & $5.2 \times 10^{-9} / T_{e}$ & {$[19]$} \\
(R6) & $e+O \rightarrow O^{+}+2 e$ & $9.0 \times 10^{-10} T_{e}^{0.7} \exp \left(-13.6 / T_{e}\right)$ & {$[19]$} \\
\hline
\end{tabular}

It is well known that the broadenings of emission line profiles are the consequences from the contribution of different interaction mechanisms of the particles concerned. The Doppler broadening $\left(\Delta \lambda_{\text {Doppler }}\right)$ and the instrumental broadening $\left(\Delta \lambda_{\text {inst }}\right)$ produce a profile that appears to as the Gaussian profile $(G(\lambda))$. On the other hand, the natural $\left(\Delta \lambda_{\text {nat }}\right)$, resonance $\left(\Delta \lambda_{\text {res }}\right)$, Van der Waals $\left(\Delta \lambda_{v d w}\right)$, and Stark $\left(\Delta \lambda_{\text {stark }}\right)$ broadenings can be approximated by Lorentzian profiles (Laux et al. 2003) (L $(\lambda)$ ). Thus, each broadened line contains Doppler and Stark broadenings so that the Voigt function $(B(\lambda))$ is the convolution of the Gaussian and Lorentzian functions (Ionascu-Nedecescu et al. 2008). The Gaussian and Lorentzian line emission for full width at half maximum (FWHM) can be written as (Belostotskiy et al. 2010, Gil et al. 2007),

$$
\Delta \lambda_{G}=\sqrt{\Delta \lambda_{\text {Doppler }}^{2}+\Delta \lambda_{\text {inst }}^{2}}
$$

and

$$
\Delta \lambda_{\mathrm{L}}=\Delta \lambda_{\text {Stark }}+\Delta \lambda_{v d w}+\Delta \lambda_{\text {res }}+\Delta \lambda_{\text {nat }},
$$

respectively. The $\Delta \lambda_{\text {inst }}$ is the resolution of the spectrometer taken into consideration in the analysis. For example, from the Voigt fit as shown in Fig. 5, the FWHM of Gaussian profile $\Delta \lambda_{G}=1.50002 \mathrm{~nm}$ is obtained and then using Eq. (2) $\Delta \lambda_{\text {Doppler }}=0.0068 \mathrm{~nm}$ is determined. In a similar way, the Stark broadening $\Delta \lambda_{\text {Stark }}=0.0245 \mathrm{~nm}$ is obtained from the Lorentzian profile using Eq. (3) to determine electron density. 


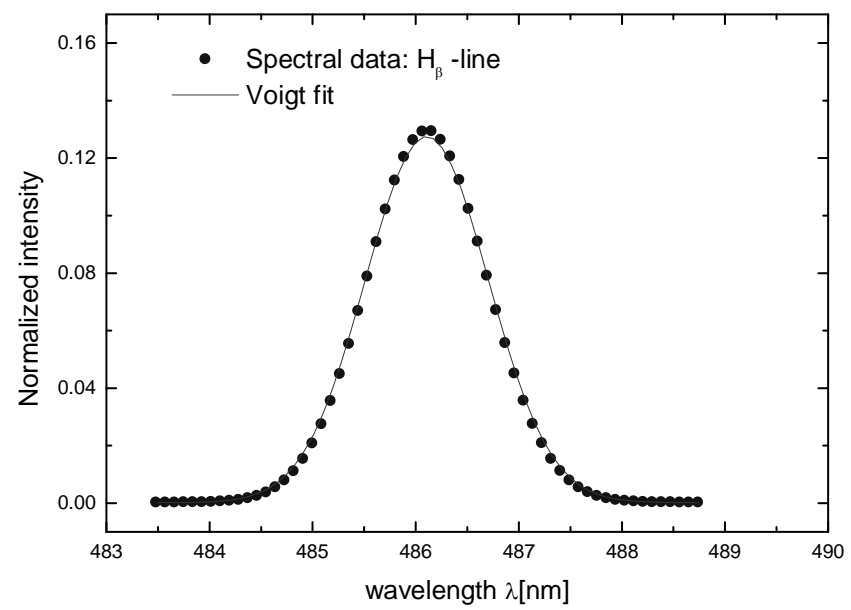

Fig. 5. Voigt function fitting of the spectroscopic data for $\mathrm{H}_{\beta}$-line $(486.12 \mathrm{~nm})$ measured at $2.5 \mathrm{kV}$ with $\mathrm{O}_{2}$ flow rate of $3 \mathrm{lpm}$.

However, $T_{g}$ is determined from the Doppler broadening $\left(\Delta \lambda_{\mathrm{G}}\right)$ by the following equation (Huddlestone et al. 1965)

$$
\Delta \lambda_{\text {Doppler }}=7.16 \times 10^{-7} \cdot \lambda_{0} \sqrt{\frac{T_{g}}{M}}(\mathrm{~nm})
$$

where $\lambda_{0}$ is the central wavelength $(\mathrm{nm}), \mathrm{M}$ is the atomic mass $\left(\mathrm{g} \cdot \mathrm{mol}^{-1}\right)$ of the emitter and $T_{g}$ is the gas temperature $(\mathrm{K})$.

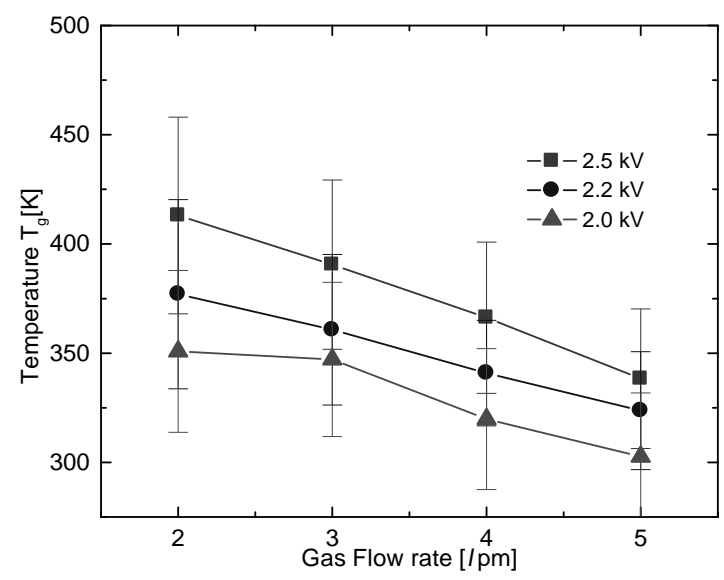

Fig. 6. Dependence of gas temperature $T_{g}$ on $O_{2}$ gas flow rate and voltage.

It is important to infer the appropriate gas temperature for biological application of the APDJ, as mentioned earlier. In the present study, emission of $H_{\beta}(4 d-2 p)$ line at 
$486.12 \mathrm{~nm}$ is used for the determination of $T_{g}$. The upper and lower limits of temperatures are estimated from the data provided by the fitting software. The estimated errors are found in the range from $15 \%-25 \%$.

Fig. 6 shows the effect of gas flow rate on $T_{g}$ at different discharge voltages. It is observed that $T_{g}$ is decreasing with increasing gas flow rate. Gas flow plays a significant role in carrying Joule heat (Raizer et al. 1991) out from the discharge region. The reason of decreasing $T_{g}$ arises due to the fact that the transit time of the oxygen species becomes shorter within the electrode gap where strong electric field exists. The electrons gain energy from the electric field in this gap and releasing energy to gas species through electron-neutral momentum transfer collision process. According to our experimental conditions when the gas flow rate is lower than $2 \mathrm{lpm}$, heat is dominantly removed by conduction ( $\mathrm{Li}$ et al. 2009) through the capillary tube wall. While the gas flow rate is greater than $2 \mathrm{lpm}$, the effect of gas flow on cooling the $T_{g}$ becomes dominant through convection process which is proportional to the gas flow velocity. Hence $T_{g}$ decreases with increasing gas flow rate. On the other hand, $T_{g}$ increases with voltage, because electrons are collecting more energy from the increased electric field and transferring this energy to neutral particles through electron-neutral momentum transfer collision process. Thus, $T_{g}$ increases with increasing voltage but decreases with increasing gas flow rate. This result is found similar to that obtained by Ionascut-Nedelcescu et al. (2008) and Li et al. ( Li 2009).

Electron density: Interactions of electrons with ions are responsible (Moravej et al. 2006) for Stark broadening, but electrons play significant role due to their lower masses and higher relative velocities. During OES data analyses, it is obtained that the contributions of $\Delta \lambda_{\text {nat }}$ and $\Delta \lambda_{\text {res }}$ are found negligible, but the dependence of $\Delta \lambda_{v d w}$ is on average $30 \%$ on $\Delta \lambda_{L}$. Thus, the value of $\Delta \lambda_{v d w}$ is subtracted from $\Delta \lambda_{\mathrm{L}}$ in order to obtain $\Delta \lambda_{\text {stark }}$. However, the simplified form for the determination of $n_{e}$ using $H_{\beta}$ line profile is expressed as (Huddlestone et al. 1965)

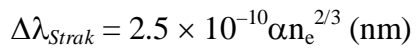

where $n_{e}$ is in $\mathrm{cm}^{-3}$ and $\alpha$ is the ion broadened parameter. The values of $\alpha$ are obtained from the least square curve fitting of the Voigt profiles and found consistent with the tabulated data of Griem et al. (Griem 1964).

Figure 7 shows the effects of voltage and $\mathrm{O}_{2}$ gas flow rate on electron density $n_{e}$. With increasing voltage, the electrons gain more energy from the enhanced electric fields and the ionizing collisions between electrons and neutrals become more frequent. Alternatively, the higher the value of electron temperature, the more effectively ionizes the neutrals causing abundant production of electrons. Therefore, most of the energies of 
the electrons, as gained from the electric field, being transferred to the oxygen species within the electrode gap through ohmic heating (Moon et al. 2002) and thereby producing more electrons and consequently $n_{e}$ increases with increasing voltage at constant $\mathrm{O}_{2}$ flow rate. On the other hand, the electron density increases as the $\mathrm{O}_{2}$ gas flow rate increases. Since oxygen is an electronegative gas and have relatively low threshold excitation level. As mentioned earlier that a large number of oxygen atoms are produced in the constricted region of the jet nozzle with the increased gas flow rate. Due to the existence of strong electric field in the jet nozzle region, the energetic electrons collided with atomic oxygen species through reactions R3, R4 and R6 (Lieberman et al. 1994, Moravej et al. 2006) and produce a huge number of electrons with increased gas flow rate.

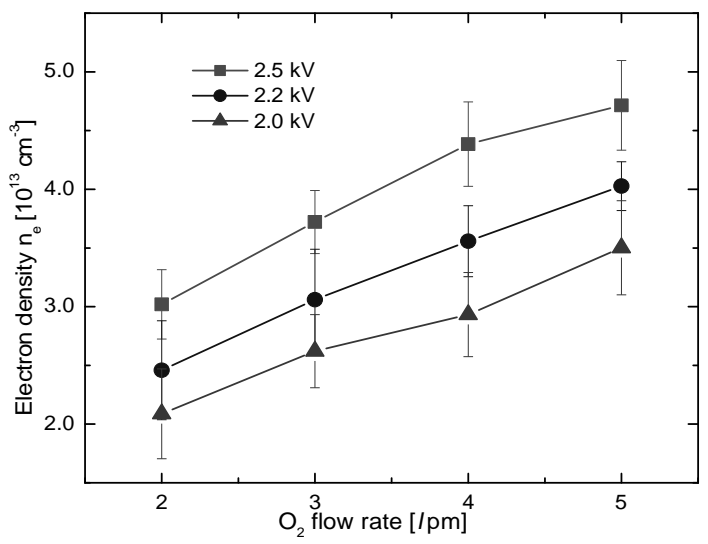

Fig. 7. Dependence of electron density $n_{e}$ on $O_{2}$ gas flow rate and voltage.

Furthermore, it is also evidenced from the recorded spectra of Fig. 3 that a significant number of positive oxygen ionic species are created which is an indication of the increased electron density. This dependence of electron density on gas flow rate is similar to that described by Moon et al. (2002), but is different from the findings of other atmospheric pressure plasma experiments (Alvarez et al. 2004).

Excitation temperature is considered as the electron temperature in atmospheric pressure plasma (Capitelli et al. 2000). For the determination of $T_{\text {exc }}$, Boltzmann plot is a simple and widely used method. In this method, the atomic transitions having large energy difference between the upper level $\left(E_{j}\right)$ and the ground level are considered. Assuming that the atomic level population follows Boltzmann distribution, the emission intensity $I_{j i}$ can be expressed (Ovsyannikov and Zhukov 2005) as

$$
I_{j i}=\frac{h c n_{j} A_{j i} g_{j}}{4 \pi Z \lambda_{j i}} \exp \left[-\frac{E_{j}}{k_{B} T_{e x c}}\right]
$$


where, $\lambda_{j i}, A_{j i}, g_{j}, k_{B}, h, c, n_{j}$ and $Z$ are the wavelengths of the emitted light, transition probability of the level considered, statistical weight of upper level, Boltzmann constant, Planck's constant, speed of light, total population density and partition function, respectively. The Boltzmann plot for $\ln \left(I_{j i} \lambda_{j i} / A_{j i} g_{j}\right)$ is drawn as a function of $E_{j}$ introducing the parameter values required along with the measured intensity $I_{j i}$. $T_{\text {exc }}$ can be determined from the slope of the linear fit. The upper and lower limits of $T_{\text {exc }}$ can be estimated in the similar way as mentioned earlier.

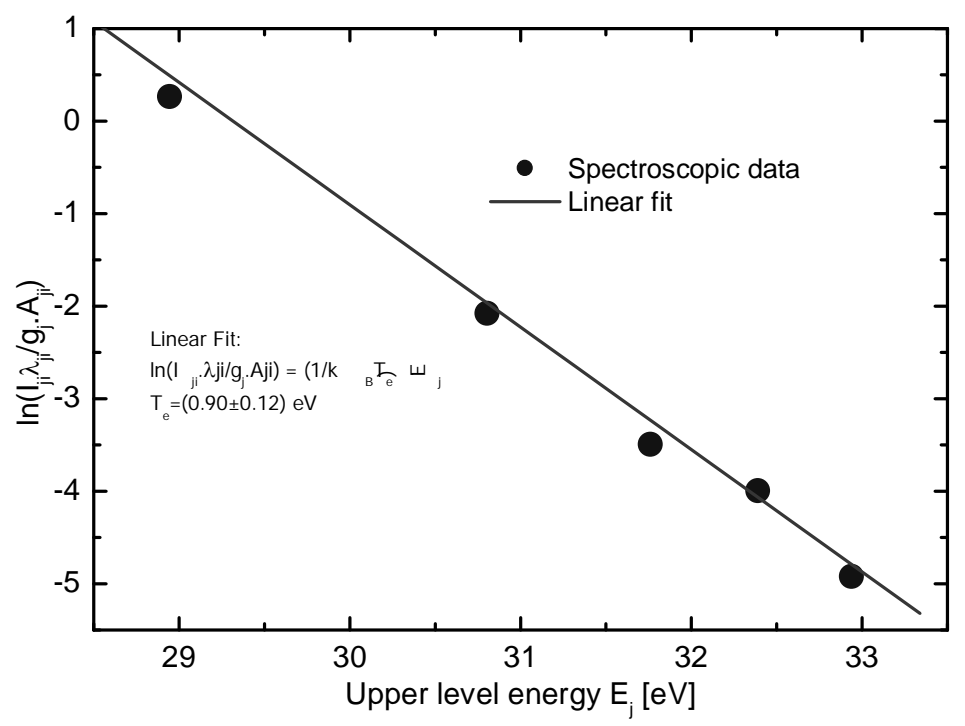

Fig. 8. Determination of excitation temperature, $T_{\text {exc }}$ by Boltzmann plot method using $\mathrm{O}^{+}$lines measured at $2.5 \mathrm{kV}$ with $\mathrm{O}^{2}$ flow rate of $3 \mathrm{lpm}$.

In this experiment, the spectral lines of $\mathrm{O}^{+}$at their emission wavelengths are selected. The transition parameters required for the determination of $T_{\text {exc }}$ are collected from the NIST database (NIST). The Boltzmann plot and a linear fit are performed as shown in Fig. 8, for an example. The effect of gas flow rate on $T_{\text {exc }}$ for different voltages is displayed in Fig. 9. It is observed that $T_{\text {exc }}$ decreases with increasing gas flow rate. This can be attributed with the Reynolds number as mentioned earlier. In one hand, with increasing gas density, the mean free path is reducing that is electron-neutral collision frequency is increasing and hence electron excitation temperature is decreasing with increasing gas flow rate. On the other hand, with increasing applied voltage, electric field intensity in the electrode gap increases and hence electrons collect more energy from the enhanced electric field. As a result $T_{e x c}$ increases with the increase of voltage. $T_{e x c}$ in the plasma is almost linearly proportional to the electric field intensity. Uhm et al. (Uhm 2000) contributed similar results in their experiments. 


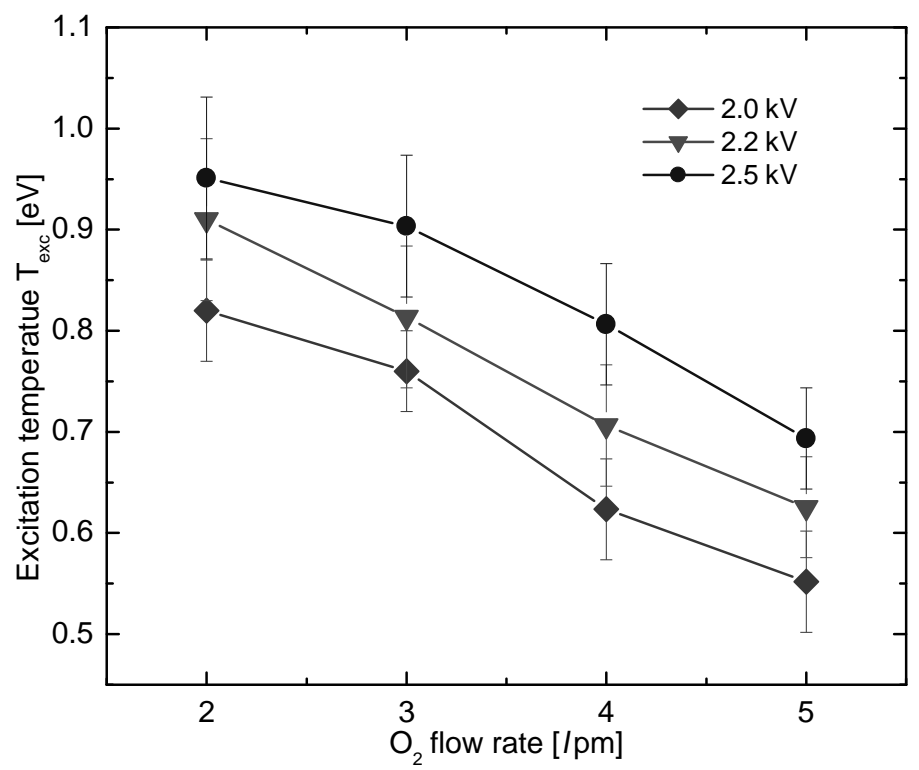

Fig. 9. Dependence of excitation temperature $T_{e x c}$ on gas flow rate and voltage.

\section{CONCLUSION}

Atmospheric pressure dielectric barrier discharge plasma jet is developed using capillary tube with oxygen as the working gas in order to generate nonthermal plasma for biological applications. Optical emission spectroscopy is used to diagnose the produced plasma. The spectral line broadening due to Doppler broadening and Stark broadening of $\mathrm{H}_{\beta}$ line is used to determine $T_{g}$ and $n_{e}$. It is found that $n_{e}$ is increases with increasing gas flow rate. $T_{e x c}$ is determined from $O^{+}$line emission employing Boltzmann plot method. It is found that $T_{g}$ decreases with increasing $O^{+}$flow rate due to shorter transit time to transfer energy through electron-neutral collisions. $T_{\text {exc }}$ is also decreasing with gas flow rate. The effect of voltage shows that all the parameters are increasing with the increase of discharge voltage. Electrical measurement reveals that the discharge frequency decreases with increasing gas flow rate, but increases with applied voltage. Finally reactive oxygen species (ROS) of APDJ is applied on Fusarium oxysporum fungus affected potato samples and it provides an initial effective result. Further study is going on to complete the expected application with this jet.

\section{ACKNOWLEDGEMENTS}

This work was supported by the Ministry of Science, Information and Communication Technology, Government of Bangladesh, under Grant No.: 39.009.002. 
01.00.020.2010/EAS-07/766 and Faculty of Engineering, University of Rajshahi, under Grants No.: 591/5/52/UGC/FE-9/2013 and 699(5)/5/52/RU/FE-4/2013.

\section{REFERENCES}

Arkhipenko, V. I., Th. Callegari, Y. A. Safronau, L. V. Simonchik and I. M. Tsuprik. 2013. Anode spot patterns and fluctuations in an atmospheric pressure glow discharge in helium. Plasma Sources Sci. Technol. 22: 045003-8.

Alvarez, R., M. C. Quintero and A. Rodero. 2004 Radial distribution of electron density gas temperature and air species in a torch kind helium plasma produced at atmospheric pressure. Spectrochim. Acta Part B $\mathbf{5 9}: 709-721$.

Belostotskiy, S. G., T. Ouk, V. M. Donnelly, D. J. Economou and N. Sadeghi. 2010. Gas temperature and electron density profiles in an argon dc microdischarge measured by optical emission spectroscopy. J. Appl. Phys. 107: 053305-7.

Capitelli, M., M. Ferreira, B. F. Gordiets and A. I. Osipov. 2000. Plasma Kinetics in Atmospheric Gases. Springer-Verlag, Berlin.

d'Agostino, R., P. Favia, C. Oehr and M. R. Wertheimer. 2005. (ed) in Plasma Processes and Polymers. Wiley, Weinheim-VCH .

Fridman, A., G. Fridman. 2013. Plasma Medicine. Wiley, UK.

Flatin, D. C., T. M. Goyette, M. M. Beaky, D. C. Ball and F. C. De Lucia. 1999. Rotational state dependence of collision induced line broadening and shift at low temperature. J. Chem. Phys. 110: $2087-2098$.

Gil, A. Y., K. Focke, J. Benedikt and A. von Keudel. 2007. Optical and electrical characterizationof an atmospheric pressure microplasma jet for $\mathrm{Ar} / \mathrm{CH}_{4}$ and $\mathrm{Ar} / \mathrm{C}_{2} \mathrm{H}_{2}$ mixtures. J. Appl. Phys. 101: $103307-8$.

Griem, H. R. 1964. Plasma Spectroscopy. McGraw-Hill, New York.

Huddlestone, R. H. and S. L. Leonard. 1965. Plasma Diagnostic Techniques. Academic Press, New York.

Ionascut-Nedelcescu, A., C. Carlone, U. Kogelschatz, D. V. Gravelle and I. M. Boulos. 2008. Calculation of the gas temperature in a throughflow atmospheric pressure dielectric barrier discharge torch by spectral line shape analysis. J. Appl. Phy.103: 063305-9.

Li, X., C. Di, P. Jia and W. Bao. 2013. Characteristics of an atmospheric-pressure argon plasma jet excited by a dc voltage. Plasma Sources Sci. Technol. 22: 045007-7.

Lieberman, L. A. and A. J. Lichtenberg. 1994. Principles of Plasma Discharges and Materials Processing. Wiley, New York.

Laux, C. O., T. G. Spence, C. H. Kruger and R. N. Zare. 2003. Optical diagnostics of atmospheric pressure air plasmas. Plasma Sources Sci. Technol. 12: 125-138.

Li, S. Z., W. T. Huang and D. Wang. 2009. The effect of gas flow on argon plasma discharge generated with a single-electrode configuration at atmospheric pressure. Phys. Plasmas 16: 093501-6.

Moravej, M., X. Yang, R. F. Hicks, J. Penelon and S. E. Babayan. 2006. A radio-frequency nonequilibrium atmospheric pressure plasma operating with argon and oxygen. J. Appl. Phys. 99: 093305-6.

Moon, S. Y., W. Choe, H. S. Uhm, Y. S. Hwang and J. J. Choi. 2002. Characteristics of an atmospheric microwave-induced plasma generated in ambient air by an argon discharge excited in an open-ended dielectric discharge tube. Phys. Plasmas 9(9): 4045-4051. 
Ni, T. L., F. Ding, X. D. Zhu, X. H. Wen and H. Y. Zhou. 2008. Cold microplasma plume produced by a compact and flexible generator at atmospheric pressure. A. Appl. Lett. 92: 241503-3.

Niemi, K., J. Waskoenig, N. Sadeghi, T. Gans and D. O'Connell. 2011. The role of helium metastable states in radio-frequency driven helium-oxygen atmospheric pressure plasma jets: measurement and numerical simulation. Plasma Sources Sci. Technol. 20: 055005-10.

Ovsyannikov, A. A. and M. F. Zhukov. 2005. Plasma Diagnostics. Cambridge International Science Publishing, UK.

Park, H. S., S. J. Kim, H. M. Joh, T. H. Chung, S. H. Bae and S. H. Leem. 2010. Optical and electrical characterization of an atmospheric pressure microplasma jet with a capillary electrode. J. Appl. Phys. 17: 033502-10.

Raizer, R. P. 1991. Gas Discharge Physics. Springer, New York.

Rusterholtz, D. L., D. A. Lacoste,G. D. Stancu,D. Z. Pai and C. O. Laux. 2013. Ultrafast heating and oxygen dissociation in atmospheric pressure air by nanosecond repetitively pulsed discharges. J. Phys. D: Appl. Phys. 46: 464010-21.

Roth, J. R. 2001. in Industrial Plasma Engineering, Vol. 2: Applications to Nonthermal Plasma Processing IOP Publishing, UK .

Stoffels, E., I. E. Kieft, R. E. J. Sladek, L. J. M. van den Bedem, E. P. van der Laan and M. Steinbuch. 2006. Plasma needle for in vivo medical treatment: Recent developments and perspectives. Plasma Sources Sci. Technol. 15: S169-180.

Thiyagarajan, M., A. Sarani and C. Nicula. 2013. Optical emission spectroscopic diagnostics of a non-thermal atmospheric pressure helium-oxygen plasma jet for biomedical applications. $J$. Appl. Phys. 113: 233302-8.

Talukder, M. R., D. Korzec and M. Kando. 2002. Probe diagnostics of high pressure microwave discharge in helium. J. Appl. Phys. 91: 9529-9538.

Uhm, H. S., E. H. Choi and G. Cho. 2000. Breakdown properties of high-pressure electrical discharge. Phys. Plasmas 7: 2744-2746.

Walsh, J. L. and M. G. Kong. 2008. Contrasting characteristics of linear-field and cross field atmospheric plasma jets. Appl. Phys. Letters 93: 111501-3.

Xiao, D., C. Cheng, J. Shen, Y. Lan, H. Xie, X. Shu, Y. Meng, J. Li and P. K. Chu. 2014. Characteristics of atmospheric-pressure non-thermal $\mathrm{N}_{2}$ and $\mathrm{N}_{2} / \mathrm{O}_{2}$ gas mixture plasma jet. J. Appl. Phys. 115: 033303-10.

Zhang, S., A. Sobota, E. M. Van Veldhuizen and P. J. Bruggeman. Gas flow characteristics of a time modulated APPJ: the effect of gas heating on flow dynamics. 2015. J. Phys. D: Appl. Phys. 48: 015203-14.

(Received revised manuscript on 7 March, 2016) 